\title{
Instrumentos para la evaluación de la sobrecarga del cuidador familiar de personas con demencia
}

\author{
África Martos, Fernando Cardila, Ana B. Barragán, Mª del Carmen \\ Pérez-Fuentes, $\mathrm{M}^{\mathrm{a}}$ del Mar Molero y José J. Gázquez \\ Universidad de Almería (España)
}

El envejecimiento poblacional en los países desarrollados es una realidad que ha dado paso al aumento de las enfermedades degenerativas, entre ellas la demencia. Los cambios que se producen en la demencia, suponen una perdida funcional que vuelve a la persona afectada más dependiente de los demás para el desempeño de las actividades, por lo que estas pasan a ser una función asumida por el cuidador. El proceso de convertirse en cuidador supone la aparición, en muchos casos de sobrecarga, esto es, la percepción que el cuidador tiene acerca de la influencia que el cuidado tiene sobre su salud, su vida social, personal y económica. La cuantificación del riesgo de agotamiento, debería ser un procedimiento común. Por ello, este estudio pretende realizar una revisión de los instrumentos de evaluación de la sobrecarga del cuidador familiar de la persona con demencia. El método que se siguió fue la recisión sistemática de las bases de datos Lilacs, ProQuest, PsycArticles y Dialnet Plus, tras la cual, se pasó a buscar la fuente primaria de los instrumentos utilizados en los estudios hallados. Así, se encontraron 4 instrumentos distintos y 6 versiones de estos. En 34 de los 42 estudios, fue utilizado como instrumento de evaluación de la sobrecarga la escala de Zarit (Zarit Caregiver Burden Interview) o una versión o adaptación de la misma. Estos resultados indican que la preferencia por esta escala para la evaluación de la sobrecarga en los cuidadores, es un continuo que no ha variado desde los años 90.

Palabras clave: Evaluación, sobrecarga, cuidador, demencia.

Instruments for evaluating the burden of family caregivers of persons with dementia. The aging population in developing countries is a reality which has given rise to degenerative diseases, among them dementia. The changes undergone during dementia cause a loss of functions which makes the person affected more dependent on others in performing their activities, and therefore these activities are taken over by the caregiver. The process of becoming a caretaker often means the appearance of overload, referring to the perception that the caregiver has of the influence that caring for that person has on their health, social and personal life and economy. Quantification of the risk of exhaustion should be done using a common procedure. Therefore, this study was intended to review instruments for evaluating the burden on family caregivers of persons with dementia. The method used was systematic review of the Lilacs, ProQuest, PsycArticles and Dialnet Plus databases, after which the primary sources of the instruments used in those studies were found. Six different versions of four different instruments were found in this way. In 34 of 42 studies, the burden evaluation instrument used was the Zarit scale (Zarit Caregiver Burden Interview) or a version or adaptation of it. These results show that the preference for this scale for evaluating burden in caregivers is continuous and without variation since the nineties.

Keywords: Evaluation, burden, caregiver, dementia.

Correspondencia: $\mathrm{M}^{\mathrm{a}}$ del Carmen Pérez Fuentes. Departamento de Psicología. Universidad de Almería. Ctra. de Sacramento s/n. C.P.: 04120. Almería (España).E-mail: mpf421@ual.es 
El envejecimiento poblacional en los países desarrollados, debido al descenso de la natalidad y de la mortalidad por los progresos socio-sanitarios, es una realidad que ha dado paso al aumento de las enfermedades degenerativas, entre ellas la demencia (Gázquez, Pérez-Fuentes, Molero, García, y Salmerón, 2011; Salmeron y Alonso, 2006). Gázquez, Pérez-Fuentes, Lucas, y Yuste (2008) encuentran una relación directa entre el aumento de la edad y la presencia de demencia. Dentro de las demencias, la enfermedad de Alzheimer es la más común entre ambos sexos (Gázquez, Pérez-Fuentes, Lucas, y Yuste, 2008; Llibre y Gutiérrez, 2014), seguida de las demencias vasculares, las demencias por cuerpos de Lewy y las degeneraciones frontotemporales (Llibre y Gutiérrez, 2014).

Los estudios epidemiológicos del último siglo apuntan a un desplazamiento de las enfermedades infecciosas, debido al aumento de la prevalencia de enfermedades no infecciosas, donde destacan los procesos neurodegenerativos (Felberg et al., 2013). La demencia es un síndrome en el que se produce una pérdida constante en áreas como la memoria, el lenguaje, las funciones ejecutivas... las cuales median en la vida cotidiana del individuo. Así, la demencia (que en la actualidad no son curables) producen un deterioro progresivo e irreversible (Llibre y Gutiérrez, 2014).

El aumento de este tipo de afecciones en la población, supone una alteración en la calidad de vida del afectado, debido a los cambios que se producen a nivel físico, social, psicológico e incluso espiritual (Escobar, 2012). Estos cambios, suponen una perdida funcional, es decir, en la capacidad de adaptarse a los problemas que surgen en lo cotidiano, de manera que el anciano con demencia necesitará ayuda del medio social y familiar para las actividades diarias por los problemas mentales y físicos que supone la enfermedad (deambulacón, malnutrición, incontinencia...) (Salmerón y Alonso, 2006). Los individuos afectados por demencia, pueden convivir 8 o 10 años con el diagnóstico, tiempo en el que la persona cada vez se vuelve más dependiente de los demás para el desempeño de las actividades (Eloah, Nitrini, y Dozzi, 2015). El deterioro paulatino de la persona con demencia, supone que las actividades de la vida cotidiana pasen a ser una función asumida por el cuidador, quien dedicara gran parte de su tiempo a atender a la persona afectada (Pérez y de la Vega, 2010). Asumir el papel de cuidador es de vital importancia en la forma de prestar las atenciones y sentirse del cuidador (Barrera et al., 2015). La demencia resulta un reto arduo en la familia del enfermo, especialmente en el cuidador principal, ya que la atención al paciente que sufre síndrome demencial, se asocia a estrés y sobrecarga, lo que se traduce en una elevada morbilidad de trastornos psicológicos en cuidadores, especialmente, depresión (Pérez y de la Vega, 2010) y estrés y somatización (Aldana y Guarino, 2012). Estos sobrevienen, en su mayoría, por la falta de preparación de los cuidadores para manejar el nuevo rol (López de Arroyabe-Castillo, 2012) o por la alta dedicación que exige el cuidado de una persona dependiente (Vázquez et al., 2014). Además, los cuidadores con demencia tienen niveles de ansiedad 
más altos que otros cuidadores de pacientes sin demencia (Eloah et al., 2015) y una sobrecarga mayor que los cuidadores formales (Aldana y Guarino, 2012).

Los cuidadores son fundamentales para evitar el internamiento de la persona con demencia, manteniéndola en la comunidad (Llibre y Gutiérrez, 215). En concreto, el cuidador principal de la persona dependiente (normalmente, familiar o amigo del mismo) sería aquel que participa en mayor medida para suministrar y organizar la atención a aquel (Guerra, Ferri, Fonseca, Banerjee, y Prince, 2011; Holosbach y Soares, 2010). Siguiendo a lo asumido por Gallardo, Barón, y Cruz (2012), aunque ellos lo establecen como definición inconclusa, "cuidado es el proceso que tiene lugar por la necesidad de una persona de ser cuidada (receptor de cuidados) y la presencia de otra que prodiga los cuidados (cuidador/a), que puede transitar desde el acompañamiento en actividades complejas hasta la dependencia para actividades básicas, siendo el cuidado informal aquel en el cual las tareas del cuidado se realiza por personas que no disponen de capacitación, no son remunerados por su tarea y tienen un elevado grado de compromiso hacia la misma, la cual es caracterizada por el afecto" (p. 90).

En cuanto al perfil del cuidador, sigue perpetuándose el modelo tradicional, donde la mujer es la principal cuidadora. En especial, cuando se trata de cuidador informar, los vínculos familiares establecen a la mujer como el sexo predominante en el cuidado a lo largo de todas las etapas vitales, bien como madre de un hijo dependiente, esposa o hija de una persona con discapacidad funcional o hija de padres de edad avanzada dependientes (Abellán, Esparza, y Pérez, 2011). Así, el perfil del cuidador prototipo sería mujer, casada, ama de casa, con hijos y un nivel de formación medio-bajo (Cerquera, Granados, y Buitrago, 2012; Escobar, 2012; Martínez, 2012; Rodríguez y Tello, 2015).

El proceso de convertirse en cuidador causa confusión en las familias, en especial al principio, debido a la alteración de roles y estilos de vida (Meyer et al., 2015). Cuando el rol de cuidador que se asume, es el del cuidador principal, la persona se enfrenta a una situación de estrés crónico (Knight y Sayegh, 2010). La carga del cuidador es la unión del miedo (a fracasar en su rol, a verse afectado en su forma de ser por la situación, a equivocarse en sus decisiones, a molestar a otros familiares, a ser testigos del dolor y sufrimiento de la persona a la que asisten) y problemas de salud, psicológicos, sociales y económicos (Lobst et al., 2009). Zarit, Reenver, y Bach-Petersen, (1980) definen la sobrecarga como la percepción que el cuidador tiene acerca de la influencia que el cuidado tiene sobre su salud, su vida social, personal y económica.

En relación al nivel de sobrecarga en los cuidadores, distintas variables han sido evaluadas a fin de conocer su influencia sobre dicho nivel, encontrando que los cuidadores mayores presentan niveles más altos, mientras que las personas con menos nivel educativo, informan de menos carga (Schindler, Engel, y Rupprecht, 2012). Al 
igual que en el estudio anterior, Kim, Knight, y Longmire (2007) encontraron en una muestra de etnia afroamericana, menores niveles de sobrecarga que relacionaron con el menor nivel educativo de la misma. Los resultados acerca de la gravedad de la demencia, apuntan que esta no afecta a la sobrecarga (Schindler et al., 2012). La relación parental con el enfermo, se asociad a la no sobrecarga, ya que los hijos asumen la tarea de cuidado del padre o madre como una tarea normal (Cerquera et al, 2012). Por otro lado, en cuando a la duración de los cuidados, los resultados son contradictorios, ya que mientras Schindler et al (2012) apuntan que el nivel de sobrecarga es independiente de la duración de la atención, Méndez, Giraldo, Aguirre-Acevedo, y Lopera (2010).encuentran que la sobrecarga si se relaciona negativamente con el tiempo de cuidado (a más tiempo, menos sobrecarga).

La cuantificación del riesgo de agotamiento, debería ser un procedimiento común, ya que la literatura ha demostrado que la carga de los cuidadores es una variable a tener en cuanta dentro del proceso terapéutico a fin de evitar en la medida de lo posible el desarrollo de enfermedades en estos (Settineri, Rizzo, Liotta, y Mento, 2014). Por ello, este estudio pretende realizar una revisión de los instrumentos de evaluación de la sobrecarga del cuidador informal de la persona con demencia.

\section{METODOLOGÍA}

Se realizó una búsqueda sistemática en las bases de datos Dialnet Plus, PsycArticles, LILACS y ProQuest. En la primera, se introdujeron los descriptores "sobrecarga del cuidador" y "demencia". Además, se aplicaron tres filtros para la selección de artículos de revista que tuviesen acceso al texto completo. No se introdujeron filtros de tipo temporal, pues la pretensión era recoger el mayor número de escalas que evaluasen la sobrecarga del cuidador de personas con algún tipo de demencia. Así, la búsqueda devolvió un total de 7 resultados. Los criterios de exclusión tras la primera lectura de los artículos fueron: que los cuidadores fueran formales, que la enfermedad del receptor de los cuidados fuese distinta a algún tipo de demencia y que no se evaluase directamente la sobrecarga del cuidador en el estudio. En relación al primer criterio (el que hace referencia a la selección de artículos con cuidadores informales) uno de los estudios contaba con una muestra de ambos tipos (cuidadores formales y no formales), por lo que no se descartó el estudio completo, contemplando solo aquellos datos que hacían referencia a los cuidadores informales.

Por otro lado, dos de los artículos devueltos en la búsqueda, no permitían el acceso al texto completo, por lo que fueron eliminados, quedando un total de 5 artículos.

En la base de datos PsycArticles, se introdujeron los descriptores "burden caragiver" and "dementia". Esto se debió a que, con antelación, se habían usado los mismos descriptores que en la búsqueda anteriormente comentada en Dialnet Plus, pero 
dado el idioma de la base de datos (concretamente, inglés), la búsqueda no devolvió ningún resultado, lo que se tradujo en la introducción de los descriptores en el mismo idioma de la base de datos. En esta búsqueda se implantaron filtros referentes a tipo de fuente (se seleccionaron revistas científicas), tipo de publicación (escogiendo entonces artículos) y disponibilidad de texto completo. No se introdujeron límites temporales por el motivo comentado anteriormente. Así, la búsqueda devolvió un total de 524 resultados, número que hubo de reducirse escogiendo que los descriptores apareciesen en el resumen. Tras esto, el número de artículos que se halló en la búsqueda fue de 16 . Al igual que en la búsqueda anterior, tras una primera lectura de los manuscritos, se aplicaron los criterios de exclusión expuestos (cuidador formal, paciente con enfermedad distinta de demencia y no evaluación directa de la sobrecarga del cuidador). Con ello, fueron eliminados 4 artículos por no realizar pruebas de evaluación de sobrecarga y uno debido a que no especificaba el diagnóstico de los pacientes, comentando solo que los pacientes sufrían "situaciones médicas complejas". Quedando en total 11 artículos.

Tabla 1. Bases de datos, descriptores, filtros y criterios aplicados en la búsqueda

\begin{tabular}{|c|c|c|c|c|c|}
\hline Base de datos & Descriptores & Filtros aplicados & $\begin{array}{c}\mathrm{N}^{\circ} \text { de } \\
\text { artículos } \\
\text { inicial }\end{array}$ & Criterios de exclusión & $\begin{array}{l}\mathrm{N}^{\circ} \mathrm{de} \\
\text { artículos } \\
\text { final }\end{array}$ \\
\hline Dialnet Plus & $\begin{array}{l}\text { "sobrecarga del } \\
\text { cuidador" y } \\
\text { demencia }\end{array}$ & $\begin{array}{l}\text {-Tipo de publicación: } \\
\text { artículos } \\
\text {-Fuente: revista científicas } \\
\text {-Acceso a texto completo }\end{array}$ & 7 & $\begin{array}{l}\text {-Población de cuidadores } \\
\text { formales. } \\
\text {-enfermedad del receptor } \\
\text { distinta de demencia } \\
\text {-no evaluación directa de } \\
\text { la sobrecarga del cuidador. }\end{array}$ & 5 \\
\hline PsycARTICLES & $\begin{array}{l}\text { "burden } \\
\text { caragiver" and } \\
\text { "dementia" }\end{array}$ & $\begin{array}{l}\text {-Descriptores en resumen } \\
\text {-Fuente: revistas } \\
\text { científicas } \\
\text { tipo de publicación } \\
\text { artículos } \\
\text {-Acceso a texto completo }\end{array}$ & 16 & $\begin{array}{l}\text {-Población de cuidadores } \\
\text { formales. } \\
\text {-enfermedad del receptor } \\
\text { distinta de demencia } \\
\text {-no evaluación directa de } \\
\text { la sobrecarga del cuidador. }\end{array}$ & 11 \\
\hline LILACS & $\begin{array}{l}\text { "sobrecarga del } \\
\text { cuidador" y } \\
\text { demencia }\end{array}$ & $\begin{array}{c}\text {-Descriptores en resumen } \\
\text {-Fuente: revistas } \\
\text { científicas } \\
\text { tipo de publicación } \\
\text { artículos } \\
\text {-Acceso a texto completo }\end{array}$ & 11 & $\begin{array}{l}\text {-Población de cuidadores } \\
\text { formales. } \\
\text {-enfermedad del receptor } \\
\text { distinta de demencia } \\
\text {-no evaluación directa de } \\
\text { la sobrecarga del cuidador. }\end{array}$ & 7 \\
\hline ProQuest & $\begin{array}{c}\text { (burden } \\
\text { caregiver OR } \\
\text { sobrecarga del } \\
\text { cuidador) AND } \\
\text { (dementia OR } \\
\text { demencia) } \\
\end{array}$ & $\begin{array}{l}\text {-Descriptores en título } \\
\text {-Fuente: revistas } \\
\text { científicas } \\
\text { tipo de publicación } \\
\text { artículos } \\
\text {-Acceso a texto completo }\end{array}$ & 29 & $\begin{array}{l}\text {-Población de cuidadores } \\
\text { formales. } \\
\text {-enfermedad del receptor } \\
\text { distinta de demencia } \\
\text {-no evaluación directa de } \\
\text { la sobrecarga del cuidador. }\end{array}$ & 19 \\
\hline
\end{tabular}

También, se realizó una búsqueda en la base de datos LILACS, donde se introdujeron los descriptores "sobrecarga del cuidador" y demencia. Además, se seleccionó que ambos descriptores apareciesen en el resumen. Los filtros aplicados fueron: que fuesen artículos con textos completos, en español o inglés. De este modo, se obtuvieron un total de 11 artículos que se pasó a revisar y que, al igual que en las 
búsquedas anteriores, fueron mediados por los criterios de exclusión siguientes: que los cuidadores evaluados fueran formales, que la enfermedad del receptor de los cuidados fuese distinta a algún tipo de demencia y que no se evaluase directamente la sobrecarga del cuidador en el estudio. En relación a estos criterios, 3 de los artículos no evaluaban la carga del cuidador. Además, dos de los resultados ya habían sido hallados en la búsqueda en la base de datos Dialnet Plus. Por lo tanto, fueron 7 los documentos finalmente seleccionados en esta base de datos.

Por último, se usó la base de datos ProQuest, en la cual se utilizaron como descriptores "burden caregiver" y "dementia", también es español ("sobrecarga del cuidador" y demencia), mediado por operadores booleanos. Como filtros, se seleccionó que fueran artículos publicados en revistas científicas, en inglés o español, y que las palabras se encontrasen en el título. Así, se hallaron 29 resultados que tras una primera lectura, donde se siguieron los criterios de exclusión anteriormente comentados, se redujeron a 19 resultados (dado que 9 no evaluaban la sobrecarga del cuidador y 1 de los artículos versaban sobre cuidadores formales).

El número final de artículos que fueron seleccionados para su revisión fue de 42. Los artículos seleccionados utilizaban escalas para la evaluación de la sobrecarga del cuidador informal de la persona con demencia. A partir de la bibliografía de estos, pasamos a buscar la fuente primeria de dichas escalas.

\section{RESULTADOS}

A partir de la lectura de los documentos seleccionados en la revisión, se hallaron 4 instrumentos originales y 5 versiones de los cuales, como se ha comentado anteriormente, se buscó la fuente primaria. Estos instrumentos se encuentran recogidos en la tabla 2 .

Zarit Caregiver Burden Interview (ZBI) es la escala que se repitió más en su uso, en concreto, en 34 de los artículos que se revisaron, y en otros 4 se utilizaron adaptaciones o versiones de esta. Tan solo en 4 de los artículos no se usó este instrumento o una variación del mismo. El ZBI se constituye originariamente como una entrevista para la evaluación de cuidadores primarios de pacientes con demencia, entrevista que fue realizada a 29 cuidadores y que obtuvo sus ítems a partir de las áreas más frecuentemente mencionada por los cuidadores (según la propia experiencia de los autores): salud del cuidador, bienestar psicológico, finanzas, vida social y relaciones sociales. Está compuesta por 29 ítems, a los que los cuidadores responden eligiendo la frase más adecuada entre "no del todo" y “extremadamente" (Zarit et al., 1980). 
Tabla 2. Instrumentos de evaluación de la sobrecarga en cuidadores principales de persona con demencia

\begin{tabular}{|c|c|c|c|c|}
\hline $\begin{array}{l}\text { INSTRUMENTO, } \\
\text { AUTORES Y ANO }\end{array}$ & $\begin{array}{l}\text { ADAPTACIONES } \\
\text { HALLADAS }\end{array}$ & $\begin{array}{l}\text { TIPO DE APLICACIÓN Y } \\
\text { CONTENIDO }\end{array}$ & $\begin{array}{c}\mathrm{N}^{\circ} \text { DE ITEMS Y } \\
\text { FORMATO DE } \\
\text { RESPUESTA } \\
\end{array}$ & $\begin{array}{l}\text { PRUEBAS } \\
\text { PSICOMË- } \\
\text { TRICAS } \\
\end{array}$ \\
\hline $\begin{array}{l}\text { Zarit Caregiver } \\
\text { Burden Interview } \\
\text { (ZCBI). } \\
\text { Zarit et al. (1980) }\end{array}$ & $\begin{array}{c}\text {-Español (Martín et al, 1996) } \\
\text {-Alemán (Zarit, Orr, y Zarit, } \\
\text { 1985) } \\
\text {-Versión corta de } 7 \text { ítems } \\
\text { ( Zarit, Todd, y Zarit, 1986) } \\
\text {-Versión corta de } 10 \text { ítems } \\
\text { (ZBI-short) (Bedard et al., } \\
\text { 2001) }\end{array}$ & $\begin{array}{l}\text { Autoadministrada o } \\
\text { heteroaplicada. Subescalas: } \\
\text { salud del cuidador, } \\
\text { bienestar psicológico, } \\
\text { finanzas, vida social y } \\
\text { relaciones sociales }\end{array}$ & $\begin{array}{l}22 \text { ítems. Likert } 5 \\
\text { puntos }\end{array}$ & $\begin{array}{c}\text { Consistencia } \\
\text { interna } 0.91 . \\
\text { Test-retest } \\
0.86 .\end{array}$ \\
\hline $\begin{array}{l}\text { Caregiver Appraisal } \\
\text { Inventory (CAI). } \\
\text { Lawton, Kleban, } \\
\text { Musgo, Ruinas, y } \\
\text { Glicksman (1989) }\end{array}$ & - & $\begin{array}{c}\text { Autoadministrada. } \\
\text { Subescalas (a) sobrecarga } \\
\text { subjetiva, (b) satisfacción } \\
\text { con el cuidado, (c) } \\
\text { percepción del impacto en } \\
\text { la vida social dominio del } \\
\text { cuidado, d) dominio del } \\
\text { cuidado } \\
\end{array}$ & $\begin{array}{l}21 \text { ítems. Escala } \\
\text { tipo likert de } 4 \\
\text { puntos }\end{array}$ & $\begin{array}{l}\text { Alfa de } \\
\text { Cronbach } \\
\text { aceptable. }\end{array}$ \\
\hline $\begin{array}{l}\text { Revised Memory } \\
\text { and Behavior } \\
\text { Problem Checklist } \\
\text { (RMBPC) Teri et } \\
\text { al., 1992) }\end{array}$ & - & $\begin{array}{l}\text { Autoadministrada por el } \\
\text { cuidador. } \\
\text { Problemas de memoria del } \\
\text { enfermo y reacción o } \\
\text { angustia del cuidador }\end{array}$ & $\begin{array}{l}24 \text { ítems. Escala } \\
\text { tipo likert de } 5 \\
\text { puntos. }\end{array}$ & $\begin{array}{c}\text { Alpha de } \\
\text { Cronbach: } .84 \\
\text { para el } \\
\text { comportamien } \\
\text { to del paciente } \\
\text { y. } 90 \text { para la } \\
\text { reacción } \\
\text { cuidador } \\
\end{array}$ \\
\hline $\begin{array}{l}\text { Caregiver Burden } \\
\text { Inventory (CBI) } \\
\text { Novak y Guest } \\
(1989)\end{array}$ & -Coreana (Kwon, 1994) & $\begin{array}{c}\text { Autoadministrado o } \\
\text { heteroadministrado. } \\
5 \text { subescalas: (a) Carga } \\
\text { temporal; (b) Carga del } \\
\text { desarrollo; (c) Carga } \\
\text { psicológica; (d) Carga } \\
\text { social; (e) Carga emocional; }\end{array}$ & $\begin{array}{l}24 \text { ítems. Likert } 5 \\
\text { puntos }\end{array}$ & $\begin{array}{c}\text { Alpha de } \\
\text { Cronbach: } .85\end{array}$ \\
\hline
\end{tabular}

The Revised Memory and Behavior Problems Checklist es un instrumento diseñado para la evaluación de los problemas de conducta en pacientes con demencia, aunque en el estudio inicial, la muestra estaba compuesta por 201 pacientes y sus cuidadores, que acudieron a un centro médico (por lo que la muestra incluía tanto a pacientes con demencia como sin ella). Ello implica que, tal y como exponen los autores, el instrumento puede ser utilizado con fines de evaluación médica, cognitiva o psicológica en cualquier persona mayor. Contiene dos subescalas: una de ellas se centra en los comportamientos observables del paciente y otra en las reacciones del cuidador. En cuanto a sus características, esta prueba autoadministrada está conformado por 24 ítems, que el cuidador debe responder en una escala tipo likert de 5 puntos. La prueba es fiable y válida, con un alpha de .84 para la subescala de comportamiento del paciente y .90 para la reacción del cuidador. La validez concurrente y discriminante ha sido confirmada mediante la comparación con índices bien establecidos de depresión, deterioro cognitivo y la carga del cuidador (Teri et al., 1992). 
Caregiver Appraisal Inventory (CAI) se obtuvo a partir de dos proyectos distintos, uno en el que se realizó un programa de respiro para cuidadores de personas con demencia, y otro al que llamaron "estudio de institucionalización" y en el que siguieron el proceso de cuidados en el tiempo transcurrido entre la solicitud de la familia para la admisión en una institución y los primeros meses de institucionalización. Está formado por 21 ítems (dos para la evaluación del dominio del cuidado, 10 para la carga subjetiva, 5 para la satisfacción o aspectos positivos del cuidado y cuatro acerca del impacto) En cuando a las propiedades psicométricas, la consistencia interna medida mediante Alfa de Cronbach es aceptable en todos las subescalas (tal y como exponen los propios autores, quienes no establecen datos numéricos al respecto) (Lawton, Kleban, Musgo, Ruinas, y Glicksman, 1989)

Para la construcción del instrumento Caregiver Burden Inventory (CBI), se utilizó una muestra de 107 cuidadores de personas con demencia senil, Alzheimer o síndrome orgánico. El CBI establece un modelo multidimensional de la sobrecarga, siguiendo 5 factores de esta (en relación a las restricciones del tiempo del cuidador, a los sentimientos de "estar fuera" respecto a sus pares, bienestar psicológico, carga social y emocional). Está formado por 24 ítems que el cuidador responde siguiendo una escala del 0 (en absoluto) al 4 (muy descriptivo). En cuanto a las propiedades psicométricas, en el factor 1 y 2 (carga del tiempo y del desarrollo, respectivamente), obtuvieron un Alpha de Cronbach de .85 en cada uno; en el factor 3 (carga psicológica) fue de .86; en el 4(carga social), .73; y en el del 5 (carga emocional) era de .77 (Novak y Guest, 1989).

\section{CONCLUSIONES}

En 34 de los 42 resultados hallados en la búsqueda, la escala utilizada para la evaluación de la sobrecarga del cuidador informal de la persona con demencia, fue la ZBI (Zarit et al., 1980). Estos resultados indican que la preferencia por esta escala para la evaluación de la sobrecarga en los cuidadores, es un continuo que no ha variado desde los años 90, época en la que Dillehay y Sandys (1990) ya establecieron esta situación. Son múltiples las adaptaciones de esta escala, entre ellas las comentadas durante la búsqueda y recogidas en la tabla 2. Así, algunas de estas adaptaciones serían la adaptación al español de Martín et al. (1996), al alemán de Zarit, Orr, y Zarit (1985), una versión corta de 7 ítems de Zarit, Todd, y Zarit (1986), otra de 10 ítems y otra de 12 (Ballesteros et al., 2012), etc. Además, existen estudios que han probado la validez de esta escala para la evaluación de la sobrecarga en cuidadores primarios de personas con una enfermedad distinta de demencia, como por ejemplo, en cuidadores primarios de niños con enfermedad crónico degenerativa (Montero, Jurado, Valencia, Méndez, y Mora, 2014) o asma (Rodríguez, Pedraza, y Acuña, 2014). 
Las personas que asumen el rol de cuidador principal pueden ver en compromiso su salud mental y bienestar psicológico, como consecuencia de la alta dedicación que exige cuidar una persona con dependencia (Vázquez et al., 2014). En cuanto a los cuidadores de personas con demencia, la mayoría de estudios asume una alta sobrecarga (Méndez et al., 2010; Pérez y Vega, 2010). Aunque estos datos son contradictorio con otros resultados arrojados por estudios donde aparece baja sobrecarga en la mayoría de cuidadores a cargo de una persona con demencia (Cerquera et al., 2012), sobretodo en comparación con cuidadores de enfermos con enfermedades como esclerosis múltiple o traumatismo craneoencefálico (Feldberg et al., 2013). Una posible causa de esta menor sobrecarga puede encontrarse en la progresividad de la enfermedad, que permite al cuidador enfrentarse y adaptarse a su rol paulatinamente (a medida que el síndrome avanza y aparecen las perturbaciones comportamentales y demás síntomas) (Shaffer, Dooley, y Williamson, 2007) y el conocimiento existente acerca de las variaciones que sufre este tipo de pacientes (Feldberg et al., 2013). La evaluación del riesgo de agotamiento en los cuidadores es necesario para evitar el desarrollo de enfermedades ligadas a la alta carga (como las comentadas en las introducción) y la baja calidad de vida de estos (Settineri et al., 2014).

Los programas de intervención, educación y apoyo para cuidadores primarios deben ser una prioridad en atención a la salud, para proporcionar una atención y seguimiento adecuado durante el proceso de cuidar (Galindo et al., 2015; Valter, Taminatoll, y Barbosa, 2013). Además, la capacitación y el apoyo a estos es un acción necesaria para evitar la desinformación y la aparición de sobrecarga, ya que la mayoría de cuidadores indica no haber recibido ayuda de su red de apoyo ni capacitación para el cuidado (Cerquera et al., 2012). Por ello, los cuidadores necesitan asesoramiento por parte de la asistencia sanitaria, debiendo ser un objetivo prioritario de esta (Salmerón y Alonso, 2006). Otra acción válida por parte de la administración sería la destinación de fondos para la formación de personal dedicado para el cuidado, así como dar estrategias a las familias que se enfrenten a esta situación (Aldana y Guarino, 2012). Y es que, el cuidado del cuidador es fundamental para la correcta realización de su labor (Llunch, Morales, Cabrera, y Betancourt, 2010).

Las limitaciones de este estudio se encuentran en la recogida parcial de escalas, pues la revisión sistemática no permitió la recogida de la totalidad de instrumentos con el fin de evaluar la sobrecarga de cuidadores de personas con demencia, pero sí los más relevantes y versionados. Este estudio puede servir a los profesionales o a los propios cuidadores para conocer las escalas existentes en la evaluación de la sobrecarga, a fin de poder afrontarla y poner medios cuando esta aparezca en el cuidador. Pues, como hemos mencionado, la sobrecarga afecta a la mayoría de aspectos de la vida del cuidador, quien, además, realiza de manera menos eficaz la atención a la persona a su cargo y puede ver comprometida su propia salud. 


\section{REFERENCIAS}

Abellán, A., Esparza, C., y Pérez, J. (2011). Evolución y estructura de la población en situación de dependencia. Cuadernos de Relaciones Laborales, 29, 43-67.

Aldana, G., y Guarino, L. (2012). Sobrecarga, afrontamiento y salud en cuidadoras de pacientes con demencia tipo Alzheimer. Summa Psicológica UST, 9(1), 5-14.

Ballestero, J., Santos, B., González-Fraile, E., Muñoz-Hermoso, P., Domínguez-Panchón, A.I., y Martín-Carrasco, M. (2012). Unidimensional 12 Item Zarit Caregiver Burden Interview for the Assessment of Dementia Caregiver's Burden Obtained by Item Responde Theory. Value in Health, 15(8), 1141-1147.

Barrera, L., Carrillo, G.M., Chaparro-Díaz, L., Sánchez, B., Vargas, E., y Carreño, S.P. (2015). Validez de constructo y confiabilidad de instrumento calidad de vida versión familiar en español. Enfermería Global, 14(1), 227-249.

Bedard, M., Molloy, D.W., Squire, L., Dubois, S., Lever. J.A., y O'Donnell, M. (2001). The Zarit Burden Interview: a new short version and screening version. Gerontologist, 41, 652 657.

Cerquera, A.M., Granados, F.J., y Buitrago, A.M. (2012). Sobrecarga en cuidadores de pacientes con demencia tipo Alzheimer. Psychologia: Avances de la Disciplina, 6(1), 35-45.

Dillehay, R.C., y Sandys, M.R. (1990) Caregivers for Alzheimer's patients: what we are learning from research. International Journal of Aging and Human Development, 30(4), 263-285.

Eloah, C., Nitrini, R., y Dozzi, S.M. (2015). Indirect cost with dementia. A Brazilian study. Dementia e Neuropsychologia, 9(1), 42-50.

Escobar, C.L. (2012). Perception of the quality of life family caregivers of adults with cancer. Investigación y Educación en Enfermería, 30(3), 320-329.

Feldberg, C., Clemente, M.A., Tartaglini, M.F., Hermida, P.D., Pereyra, C.I., y Stefani, D. (2013). Enfermedad crónica y sentimiento de sobrecarga. Un estudio descriptivo comparativo en cuidadores familiares de pacientes con diferente patología neurológica. Perspectivas en Psicología, 10, 10-17.

Galindo, O., Rojas, E., Ascencio, L., Meneses, A., Aguilar, J.L., Olvera, E., Diez, F., y Alvarado S. (2015). Guía de práctica clínica para la atención psico-oncológica del cuidador primario informal de pacientes con cáncer. Psicooncología, 12, 87-104.

Gallardo, R., Barón, D., y Cruz, E. (2012). El cuidado informal en enfermos de Alzheimer: evaluación a partir de un modelo teórico. Revista de Ciencias Médicas de Pinar del Río, 16(1), 188-199.

Gázquez, J.J., Pérez-Fuentes M.C., Lucas, F., y Yuste, N. (2008). Prevalencia de los trastornos mentales en la población mayor. Anales de Psicología, 24(2), 327-333.

Gázquez, J.J., Pérez-Fuentes, M.C., Molero, M.M., García, M.M., y Díaz, A. (2011). Incidencia de la enfermedad de Alzheimer en la población española. En M.C. Pérez-Fuentes y J.J. Gázquez (coord.), Envejecimiento y Demencia: un enfoque multidisciplinar (pp. 33-37). Grandada: GEU.

Guerra, M., Ferri, C.P., Fonseca, M., Banerjee, S., y Prince, M. (2011). Helping carers to care: the 10/66 Dementia Research Group's randomized control trial of a caregiver intervention in Peru. Revista Brasileira de Psiquiatria, 33(1), 47-54.

Holosbach, G., y Soares, T. (2010). Experiencies of caregivers of children and teenagers with cancer. Série: Ciências da Saúde, 11, 89-108.

Kim, J.H., Knight, B.G., y Longmire, C.V. (2007). The role of familism in stress and coping processes among African American and White dementia caregivers: Effects on mental and physical health. Health Psychology, 26(5), 564-576. 
Knight, B.G., y Sayegh, P. (2010). Cultural values and caregiving: The updated sociocultural stress and coping model. The Jounal of Gerontoly, 65, 5-13.

Kwon, J.D. (1994). A study on the assessment of care-giver burden in caring for the demented elderly in Korea; Graduate School of Yonsei University Collection of PhD Dissertations.

Lawton, M.P., Kleban, M., Moss, M., Rovine, M., y Glicksman, A. (1989). Measuring caregiving appraisal. Journal of Gerontology, 44, 61-71.

Llibre, J., y Gutiérrez, R.F. (2014). Demencias y enfermedad de Alzheimer en América Latina y el Caribe. Revista Cubana de Salud Pública, 40(3), 378-387.

Llunch, A., Morales, A., Cabrera, M., y Betancourt, N. (2010). Factores previsibles en la salud física y psicosocial del cuidador crucial del anciano con demencia en el hogar. Revista Cubana de Enfermería, 26(2), 3-13.

Lobst, E., Alderfer, M., Jane, O., Askins, M., Fairclough, D., Katz, E., Butler, R., Dolgin, M., y Noll, R. (2009). Problem solving and maternal distress at the time of a child's diagnosis of cancer in two-parent versus lone-parent households. Journal of Pediatric Psychology, 34, 817-821.

López de Arroyabe-Castillo, E. (2012). Evaluación de las necesidades de los familiares de personas afectadas de Daño Cerebral Adquirido mediante el Cuestionario de Necesidades Familiares. Anales de Psicología, 28(3), 728-735.

Martínez, F.E. (2012). Recuperación de la salud del cuidador principal, en proyecto sociocultural "Quisicuaba". Los Sitios, Centro Habana. Enfermería Global, 25, 92-103.

Martín, M., Salvado, S., Mijo L., Rico, J., Lanz, P. y Taussig, M. (1996). Adaptación para el medio español de la Escala de Sobrecarga del Cuidador (Caregiver Burden Interview) de Zarit. Revista Gerontología, 6(4), 338-346.

Méndez, L., Giraldo, O., Aguirre-Acevedo, D., y Lopera, F. (2010). Relación entre ansiedad, depresión, estrés y sobrecarga en cuidadores familiares de personas con demencia tipo Alzheimer por mutación e280a en presenilina 1. Revista Chilena de Neuropsicología, 5(2), 37-145.

Meyer, O.L., Hanh, K., Nhu, T., Vu, P., Arean, P., y Hinton, L. (2015). The Sociocultural Context of Caregiving Experiences for Vietnamese Dementia Family Caregivers. Asian American Journal of Psychology, 6(3), 263-272.

Montero, X., Jurado, S., Valencia, A., Méndez, J., y Mora, I. (2014). Escala de carga del cuidador de Zarit: evidencia de validez. Psicooncología, 11(1), 71-85.

Novak, M., y Guest, C.I. (1989). Application of a multidimensional caregiver burden inventory. Gerontologist, 29, 798-803.

Pedraza, A., Rodríguez, C., y Acuña, R. (2014). Análisis factorial confirmatorio de la Escala de sobrecarga del cuidador de Zarit en padres/cuidadores de pacientes pedriátricos asmáticos. IATREIA, 27(4), 361-369.

Pérez, V.T., y de la Vega, T. (2010). Repercusión de la demencia en los cuidadores primordiales del policlínico "Ana Betancourt". Revista Cubana de Medicina General Integral, 26(2), 215-224

Rodríguez, M., y Tello, P.M. (2015). Atención a las cuidadoras de pacientes oncológicos y paliativos en el espacio comunitario. Revista Enfermería, 1(103), 15-18.

Salmerón, M., y Alonso, P. (2006). Factores asociados a la institucionalización de pacientes con demencia y sobrecarga del cuidador. Revista del Ministerio de Trabajo e Inmigración, 60, 129-148.

Schindler, M., Engel, S., y Rupprecht, R. (2012). The impact of perceived knowledge of dementia on caregiver burden. GeroPsych, 25(3), 127-134.

Settineri, S., Rizzo, A., Liotta, M., y Mento, C. (2014). Caregiver's Burden and Quality of Life: International Journal of Psychological Research, 7(1), 30-39. 
Teri, L., Truax, P., Logsdon, R., Uomoto, J., Zarit, S., y Vitaliano, P.P. (1992). Assessment of Behavioral Problems in Dementia: The Revised Memory and Behavior Problems Checklist (RMBPC). Psychology and Aging, 7(4), 622-631.

Valter, C.M.M., TaminatoIII, M., y Barbosa, D.A. (2013). Effectiveness of educational programs on reducing the burden of caregivers of elderly individuals with dementia: a systematic review. Revista Latino-Americana de Enfermagem, 21, 267-275.

Vázquez, F.L., Hermida, E., Torres, A., Otero, P., Blanco, V., y Díaz, O. (2014). Eficacia de una intervención preventiva cognitivo conductual en cuidadoras con síntomas depresivos elevados. Psicología Conductual, 22(1), 79-96.

Zarit, S. H., Orr, N. K, y Zarit, J. M. (1985). Understanding the stress ofcaregivers: Planning an intervention. En S. H. Zarit, N. K. Orr, \& J. M. Zarit (Eds.), The hidden victims of Alzheimer's disease: Families under stress (pp. 69-86). New York: New York University Press.

Zarit, S.H., Reever, K.E., y Bach-Peterson J. (1980). Relatives of the impaired elderly. Correlates of feelings of burden. Gerontologist, 20, 649-654.

Zarit, S.H., Todd, P.A., y Zarit, J.M. (1986). Subjective burden of husbands and wives as caregivers: a longitudinal study. Gerontologist, 26(3), 260-266.

Recibido: 15 de mayo de 2015

Recepción Modificaciones: 1 de julio de 2015

Aceptado: 15 de julio de 2015 